\title{
COMPLEX RANDOM MATRIX MODELS WITH POSSIBLE APPLICATIONS TO SPIN-IMPURITY SCATTERING IN QUANTUM HALL FLUIDS
}

\author{
S. Hikami ${ }^{1}$ and A. Zee ${ }^{2}$ \\ Department of Pure and Applied Sciences ${ }^{1}$ \\ University of Tokyo, Meguro-ku, Komaba, Tokyo 153, Japan \\ Institute for Theoretical Physics ${ }^{2}$ \\ University of California Santa Barbara, CA 93106, USA
}

\begin{abstract}
We study the one-point and two-point Green's functions in a complex random matrix model to sub-leading orders in the large $N$ limit. We take this complex matrix model as a model for the two-state scattering problem, as applied to spin dependent scattering of impurities in quantum Hall fluids. The density of state shows a singularity at the band center due to reflection symmetry. We also compute the onepoint Green's function for a generalized situation by putting random matrices on a lattice of arbitrary dimensions.
\end{abstract}




\section{Introduction}

Recently, matrix modes have been studied in various contexts. In particular, in a series of papers [1-4], the universality of the connected two-point correlation function was discussed. These studies focussed on $N$ by $N$ Hermitian matrices with $N$ approaching infinity.

In this paper, we investigate the behavior of the Green's functions of a complex matrix model. To leading order in the large $N$ limit, complex matrix model and Hermitian matrix model behave similarly. For instance, the density of state, for Gaussian randomness, obeys Wigner's semi-circle law in both cases. However, in subleading orders, complex random matrix and Hermitian random matrix behave differently. In the language of random surfaces, a random complex matrix model represents, due to the complex conjugate pairs, a surface made of plaquets with two colors, like a red and black checkerboard, with the rule that a given plaquette can only be glued to a plaquette of a different color.

Our motivations stem from possible applications of the complex random matrix model to physical problems involving scattering on impurities. In particular, recently Hikami, Shirai, and Wegner [5] has proposed a model for impurity scattering in quantum Hall fluids in the spin degenerate case. For certain quantum Hall samples, disorder broadening can be much larger than the Zeeman splitting between spin up and spin down electrons, in which case the spin up and spin down electrons have the same energy. In [5] the further simplification is made that when a spin up electron scattering on an impurity becomes a spin down electron, and vice versa. This is known as the "strong spin orbit" case, in which case it is known that an extended state appears at the band center of the lowest Landau level with white noise Gaussian random scattering and that the density of state shows a singularity at the band center [5-9]. Although this problem has been simulated numerically [9], the nature of the singularity of the density of state remains unclear. Another example involving scattering between two states occurs in high temperature superconductors, in which the conducting plane contains two different sites, copper and oxygen. The density of state also shows a singular behavior at the band center.

More generally, the problem of scattering between $C$ sectors (with $C=2$ in the example mentioned above) is of interest. It turns out that a class of matrix models called "lattices of matrices" and studied by Brézin and Zee 
[3] is relevant for this class of problem. In these models, random matrices are placed on a lattice of arbitrary dimension [10]. It was shown that in the large $N$ limit, various correlation functions can be determined. The twostate scattering problem considered here corresponds to the simple case of a lattice consisting of two points. Following the analysis of [3] we can readily generalize the two- state scattering problem to an arbitray lattice.

In this paper, we show that the one-point Green's function is singular in the next-to-leading order in the large $N$ expansion. We first evaluate the one-point Green's function and the density of state to order $1 / N^{2}$ by the diagrammatic method for one matrix model. The singular behavior of the one-point Green's function in order $1 / N^{2}$ has been noticed in the literatures [11], but our discussion of this singularity in the context of the two-state scattering problem in condensed matter physics may be new. Next we discuss the origin of this spurious singularity by the orthogonal polynomial method.

On the other hand, if we fix $N$ to be large but finite, and let the energy $E$ go to zero, the density of state oscillates and eventually goes to zero. This phenomenon is due to energy-level repulsion. The discussion here is somewhat reminiscent of the double pole encountered in the connected correlation function in the Hermitian matrix model when evaluated in the diagrammatic approach [2]. In the diagrammatic approach, to calculate the Green's functions we select diagrams by letting $N$ go to infinity first. We then obtain the correlation function by taking the imaginary part of the two-point Green's function. The connected correlation function has a double pole as its two arguments approach each other. In contrast, in the orthogonal polynomial approach, we in effect take the imaginary part of the two-point Green's function first, and then let $N$ go to infinity. The connected two-point correlation calculated in this way does not have a double pole when its two arguments approach each other. However, when we smooth out the short distance oscillations of the correlation function by averaging it appropriately, we recover the double pole obtained in the diagrammatic approach [1].

This paper is organized as follows. In section two, the two-state scattering problem is formulated as a complex matrix model. We evaluate the one point Green's function diagrammatically and discuss the singularity of the density of state in $d=0$. In section three, we study the complex matrix model further using the orthogonal polynomial method. In section four, we develop the analysis for general dimensions, using the "lattice of matrices" formulation given in [3]. Using the diagrammatic expansion $[12,13]$, we obtain 
the expression for the one-point Green's function. We compare our results with that obtained in previous studies [14-16].

\section{Matrix model formulation of two-state scat- tering}

In the standard Hermitian one-matrix model, the one-point Green's function $G(z)$ is defined by

$$
G(z)=\frac{1}{N}<\operatorname{Tr} \frac{1}{z-\varphi}>
$$

where the average is taken with the probability distribution $P(\varphi)$

$$
P(\varphi)=\exp [-N \operatorname{Tr} V(\varphi)]
$$

The $N \times N$ matrix $\varphi$ is Hermitian. In the Gaussian case, we have

$$
V(\varphi)=\frac{1}{2} \operatorname{Tr} \varphi^{2}
$$

More generally, we have, for example, as

$$
V(\varphi)=\frac{1}{2} \operatorname{Tr} \varphi^{2}+\frac{g}{N} \operatorname{Tr} \varphi^{4}
$$

For application to disordered systems, the random matrix $\varphi$ is interpreted as the Hamiltonian.

We start with the simplest case of two-state scattering. The model is described by the random Hamiltonian [3],

$$
H=\left(\begin{array}{cc}
H_{1} & \varphi^{\dagger} \\
\varphi & H_{2}
\end{array}\right)
$$

taken from the Gaussian distribution $P(H)$,

$$
P(H)=\frac{1}{Z} e^{-N \operatorname{Tr}\left[\frac{1}{2}\left(m_{1}^{2} H_{1}^{2}+m_{2}^{2} H_{2}^{2}\right)+m^{2} \varphi^{+} \varphi\right]}
$$

The matrices $H_{1}$ and $H_{2}$ are Hermitian while $\varphi$ is complex. In [3] this Hamiltonian was taken to describe a system with two sectors $(\mathrm{C}=2)$. Here 
we can think of the two sectors as representing as the spin up and spin down sectors in a spin-dependent quantum Hall system. We may also think of possible applications to the double-layered quantum Hall system $[17,18]$.

We now go to the model of (2.6) by letting $m_{1}^{2}=m_{2}^{2}=\infty$, so that $H_{1}$ and $H_{2}$ are suppressed. This model is for the off-diagonal disorder. This same complex matrix model is considered also in [19]. (We will treat the more general case with $H_{1}$ and $H_{2}$ non- zero later in this paper.) We have

$$
H=\left(\begin{array}{cc}
0 & \varphi^{\dagger} \\
\varphi & 0
\end{array}\right)
$$

Notice that there exists a matrix

$$
\Gamma=\left(\begin{array}{cc}
I & 0 \\
0 & -I
\end{array}\right)
$$

such that

$$
\{\Gamma, H\}=0
$$

This implies that if $\psi$ is an eigenstate of $H$ with eigenvalue $E$, then $\Gamma \psi$ is an eigenstate with eigenvalue $-E$. Thus, eigenvalues of $H$ come in pairs. Due to level repulsion, around $E=0$, there should be a "hole" of width of order $1 / N$ in the density of state $\rho(E)$. As $N$ goes to infinity, this hole disappears and $\rho(E)$ should become smooth.

To proceed, we calculate the one point Green's function, with $P=$ $Z^{-1} \exp \left(-\frac{1}{2} \operatorname{tr} H^{2}\right)$ where $H$ is given by $(2.7)$.

$$
G(z)=<\frac{1}{2 N} \operatorname{Tr}\left(\frac{1}{z-H}\right)>=<\frac{1}{N} \operatorname{Tr}\left(\frac{z}{z^{2}-\varphi^{\dagger} \varphi}\right)>
$$

To leading order in the large $N$ limit, we obtain easily

$$
G(z)=\frac{z-\sqrt{z^{2}-4}}{2}
$$

using for example the diagrammatic approach of [2]. The density of state $\rho(E)=-\frac{1}{\pi} \operatorname{Im} G(E)$ is given by the semi-circle law

$$
\rho(E)=\frac{1}{2 \pi} \sqrt{4-E^{2}}
$$


We denote $(2.11)$ by $G_{0}(z)$ hereafter.

To leading order, as expected, the density of state $\rho(E)$ is smooth, without any singularity at $E=0$. However, if we go to order $1 / N^{2}$, we will find a divergent term in the one point Green's function. Henceforth, we will work with the Gaussian distribution. (We expect that the singularity at $E=0$ will occur also for a non-Gaussian distribution.)

Using the diagrammatic approach, we can readily evaluate the Green's function to order $1 / N^{2}[2,12,13]$. We decompose the self- energy $\Sigma(z)$ into two parts $\Sigma_{a}$ and $\Sigma_{b}$, obtained by breaking the solid line (quark line in the terminology of [2]) in the diagrams $D_{a}$ and $D_{b}$, respectively.

In the simplest two-state scattering case considered here, in which scattering from 1 to 2 and from 2 to 1 occurs, but not from 1 to 1 or from 2 to 2 , we see that the numbers $n$ and $m$ appearing in the diagram $D_{a}$, describing the number of rungs in the gluon ladders, must be even. In the diagram $D_{b}$, $n_{1}, n_{2}$ and $n_{3}$ must be all even, or all odd. We have from diagram $D_{a}$ two terms as follows. Denoting the even number of rungs in the ladder by $2 n$ and $2 m$, we have

$$
\begin{aligned}
\Sigma_{a} & =\sum_{n, m=1}^{\infty} G_{0}^{4 n+4 m+1}+\sum_{n, m=1}^{\infty} G_{0}^{4 n+4 m+1}(2 n-1) \\
& =\frac{2 G_{0}^{9}}{\left(1-G_{0}^{4}\right)^{3}}
\end{aligned}
$$

The factor of $(2 n-1)$ is due to the number of ways of inserting a cut inside the ladder. Similarly, for $\Sigma_{b 1}$, we have

$$
\begin{aligned}
\Sigma_{b 1} & =\sum_{n, m, l=1}^{\infty} G_{0}^{4 n+4 m+4 l+1}+\sum_{n, m, l=1}^{\infty} G_{0}^{2(2 n-1)+2(2 m-1)+2(2 l-1)+1} \\
& =\frac{G_{0}^{7}\left(1+G_{0}^{6}\right)}{\left(1-G_{0}^{4}\right)^{3}}
\end{aligned}
$$

For $\Sigma_{b 2}$, in which the cut appears inside the ladder, we have

$$
\begin{aligned}
\Sigma_{b 2} & =\sum_{n, m, l=1}^{\infty} G_{0}^{4 n+4 m+4 l+1}(2 n-1) \\
& +\sum_{n, m, l=1}^{\infty} G_{0}^{2(2 n-1)+2(2 m-1)+2(2 l-1)+1}(2 n-2)
\end{aligned}
$$




$$
=\frac{2 G_{0}^{7}\left(1+G_{0}^{6}\right)}{\left(1-G_{0}^{4}\right)^{4}}-\frac{2 G_{0}^{7}+G_{0}^{13}}{\left(1-G_{0}^{4}\right)^{3}}
$$

Thus we get

$$
\begin{aligned}
\Sigma_{b} & =\Sigma_{b 1}+\Sigma_{b 2} \\
& =\frac{2 G_{0}^{7}\left(1+G_{0}^{6}\right)}{\left(1-G_{0}^{4}\right)^{4}}-\frac{G_{0}^{7}}{\left(1-G_{0}^{4}\right)^{3}}
\end{aligned}
$$

Adding the two terms $\Sigma_{a}$ and $\Sigma_{b}$, we have

$$
\Sigma=\frac{G_{0}^{7}}{\left(1+G_{0}^{2}\right)^{2}\left(1-G_{0}^{2}\right)^{4}}
$$

where $G_{0}$ is the one-point Green's function (2.9) evaluated to leading order in the large $N$ limit. After including an extra factor $1 /\left(1-G_{0}^{2}\right)$ for the external legs, we obtain the one-point Green's function to order $1 / N^{2}$

$$
G(z)=G_{0}+\frac{1}{N^{2}} \frac{G_{0}^{7}}{\left(1+G_{0}^{2}\right)^{2}\left(1-G_{0}^{2}\right)^{5}}+O\left(\frac{1}{N^{4}}\right) .
$$

Since $G_{0}(z)$ goes to $(-i)$ as $z \rightarrow 0$, the factor $1 /\left(1+G_{0}^{2}\right)$ diverges. Using $1+G_{0}^{2}=z G_{0}$, we have

$$
G(z)=G_{0}(z)+\frac{1}{N^{2} z^{2}\left(z^{2}-4\right)^{5 / 2}}+O\left(\frac{1}{N^{4}}\right)
$$

Thus, to order $1 / N^{2}$, the Green's function $G(z)$ has a singular imaginary part $i /\left(32 N^{2} z^{2}\right)$ as $z \rightarrow 0$. This singularity is related to the reflection symmetry (parity symmetry) as mentioned earlier. The density of state diverges like $\rho(E) \rightarrow 1 /\left(32 \pi N^{2} E^{2}\right)$. Apparently, this double pole is too singular, since the integral of the density of state should be one.

We consider next the connected two-point correlation function $\rho_{2 c}(z, w)$, which may be obtained from the connected two-point Green's function $G_{2 c}(z, w)$ as shown in $[1,2]$. We have from a diagrammatic analysis [2-4],

$$
\begin{aligned}
& N^{2} G_{2 c}(z, w)=-\frac{1}{4} \frac{\partial}{\partial w} \frac{\partial}{\partial z} \log \left[1-G^{2}(z) G^{2}(w)\right] \\
= & \left(\frac{1}{G(z) G(w)}-G(z) G(w)\right)^{-2} \frac{1}{G(z) G(w)}\left(\frac{\partial G(z)}{\partial z}\right)\left(\frac{\partial G(w)}{\partial w}\right)
\end{aligned}
$$


This leads to

$$
N^{2} G_{2 c}(z, w)=\frac{1}{4\left(z^{2}-w^{2}\right)^{2}}\left[\frac{2 z^{2} w^{2}-4 z^{2}-4 w^{2}}{\sqrt{z^{2}-4} \sqrt{w^{2}-4}}-2 z w\right]
$$

The two-point correlation function $\rho_{2 c}(z, w)$ agrees with the universal behavior in the short distance $z \rightarrow w$ limit:

$$
\rho_{2 c}(z, w)=-\frac{1}{2 \pi^{2} N^{2}(z-w)^{2}}
$$

By the equation of motion method, the complex matrix model can be studied using a recursion approach order by order in $1 / N^{2}[11]$. Pole terms appear in each order of $1 / N^{2}$. The one-point Green's function diverges in the $k$-th order as

$$
\delta G(z) \sim \frac{c_{k}}{N^{2 k} z^{2 k}}
$$

Since the connected two-point correlation function in the Hermitian matrix model does not have a double pole in the orthogonal polynomial approach, let us use the orthogonal polynomial approach to investigate whether we have the same situation for the one-point Green's function $G(z)$ in the limit $z \rightarrow 0$.

\section{Orthogonal polynomial analysis}

Since $\varphi^{+} \varphi$ can be regarded as a Hermitian matrix with positive eigenvalues, we can write the joint probability distribution in terms of its positive eigenvalues $\epsilon_{i}[19,20]$

$$
P_{N}\left(\varepsilon_{1}, \ldots, \varepsilon_{N}\right) d \varepsilon_{1} \cdots d \varepsilon_{N}=C e^{-N \sum \varepsilon_{i}} \Pi_{i<j}\left(\varepsilon_{i}-\varepsilon_{j}\right)^{2} d \varepsilon_{1} \cdots d \varepsilon_{N}
$$

with $C$ a normalization constant. The relevant orthogonal polynomial is the Laguerre polynomial $L_{n}(x)$, where $L_{0}(x)=1, L_{1}(x)=1-x$, and $L_{2}(x)=1-$ $2 x+x^{2} / 2$. The density of state $\rho(\varepsilon)$ and the two-point connected correlation function

$\rho_{2 c}\left(\varepsilon, \varepsilon^{\prime}\right)$ are given in terms of the kernel $K\left(\varepsilon, \varepsilon^{\prime}\right)[1]$ as

$$
\rho(\varepsilon)=K(\varepsilon, \varepsilon)
$$




$$
\rho_{2 c}\left(\varepsilon, \varepsilon^{\prime}\right)=-\left[K\left(\varepsilon, \varepsilon^{\prime}\right)\right]^{2}
$$

where

$$
K\left(\varepsilon, \varepsilon^{\prime}\right)=\frac{1}{N} \sum_{0}^{N-1} \psi_{n}(\varepsilon) \psi_{n}\left(\varepsilon^{\prime}\right)
$$

and

$$
\psi_{n}(\varepsilon)=e^{-N \varepsilon / 2} L_{n}(N \varepsilon)
$$

Noting that $\varepsilon=E^{2}$ in our terminology, we have an extra factor of $E$ for the density of state since $d \varepsilon=2 E d E$. At $E=0$, all Laguerre polynomials are equal to one, so the density of state should vanish at $E=0$ due to this extra factor $E$ in accordance with the reflection symmetry argument given earlier.

This result for the density of state at $E=0$ seems to contradict the result we obtained in the previous section. But there is no contradiction. In the previous section, we took the large $N$ limit first, so that the density is effectively smoothed over a certain width. Here we take $N$ large but fixed and let $E$ go to zero, and obtain $\rho(E)=0$. We see the hole described earlier. This non-commutativity of the two limits discussed here is similar to the discussion of the two-point connected correlation function of a Hermitian matrix model [1, 2].

Using the Christoffel-Darboux identity, we have a compact expression for the density of state,

$$
\begin{aligned}
\rho(\varepsilon) & =e^{-N \varepsilon} \sum_{k=0}^{N-1} L_{k}^{2}(N \varepsilon) \\
& =N e^{-N \varepsilon}\left[L_{N}(N \varepsilon) L_{N-1}^{\prime}(N \varepsilon)-L_{N-1}(N \varepsilon) L_{N}^{\prime}(N \varepsilon)\right] .
\end{aligned}
$$

Since $\varepsilon=E^{2}$, the density of state $\rho(E)$ is

$$
\rho(E)=2 E e^{-N E^{2}} \sum_{k=0}^{N-1} L_{k}^{2}\left(N E^{2}\right)
$$

In Fig. 2, this density of state is shown for $N=5$ and $N=10$. There appears N-oscillations in the density of state, and the first peak near $E=0$ is finite for $N \rightarrow 0$. The ratio of the value of the first peak to the second one is almost 1.2. In Fig.2, the dotted line represents the semi-circle behavior in the large $\mathrm{N}$ limit given by $\sqrt{4-E^{2}} / \pi$. When the oscillating part is averaged 
smoothly, we obtain the correction of the density of state to the semi-circle law of order $1 / N^{2}$.

$$
\Delta \rho=<\rho(E)>-\frac{1}{\pi} \sqrt{4-E^{2}}
$$

It may be useful to write the asymptotic expression by the Bessel function. Knowing the large $N$ behavior (for $E$ small ) of Laguerre polynomials, we can write the preceding in terms of Bessel function. Remarkably, the oscillating part near $E=0$ in Fig.2 is approximated by

$$
\rho(E) \simeq 2 N E\left[J_{0}^{2}(2 N E)+J_{1}^{2}(2 N E)\right]
$$

By plotting the oscillating curve of this equation, we find that the first peak near $E=0$ is almost same as the first peak value of (3.7). The ratio of the first peak to the second one is also 1.2, which we mentioned before. Now we use Hankel's asymptotic expansion of Bessel functions $J_{0}(t), J_{1}(t)$, for the large $t=2 N E$.

$$
\begin{aligned}
& J_{0}(t)=\sqrt{\frac{2}{\pi t}}\left[P(0, t) \cos \left(t-\frac{\pi}{4}\right)-Q(0, t) \sin \left(t-\frac{\pi}{4}\right)\right] \\
& J_{1}(t)=\sqrt{\frac{2}{\pi t}}\left[P(1, t) \cos \left(t-\frac{3 \pi}{4}\right)-Q(1, t) \sin \left(t-\frac{3 \pi}{4}\right)\right]
\end{aligned}
$$

where

$$
\begin{aligned}
P(l, t) & =\sum_{k=0}^{\infty}(-1)^{k} \frac{(l, 2 k)}{(2 t)^{2 k}} \\
& \sim 1-\frac{\left(4 l^{2}-1\right)\left(4 l^{2}-9\right)}{128 t^{2}}+\cdots \\
Q(l, t) & =\sum_{k=0}^{\infty}(-1)^{k} \frac{(l, 2 k+1)}{(2 t)^{2 k+1}} \\
& \sim \frac{4 l^{2}-1}{8 t}+\cdots \\
(l, m) & =\frac{\Gamma\left(\frac{1}{2}+l+m\right)}{m ! \Gamma\left(\frac{1}{2}+l-m\right)}
\end{aligned}
$$

We use the smooth average for the oscillating part by setting $<\sin ^{2}(x)>$ $=<\cos ^{2}(x)>=\frac{1}{2}$. Then it is easy to find that the density of state becomes

$$
\rho^{\text {smooth }}(E)=\frac{2}{\pi}\left(1+\frac{1}{32 N^{2} E^{2}}-\frac{9}{2048 N^{4} E^{4}}+\cdots\right) .
$$


The term of order $1 / N^{2}$ agrees with the result obtained in the previous section by the diagrammatic method. In this approximation, the leading term is given simply by $\frac{2}{\pi}$ for $\sqrt{4-E^{2}} / \pi$. Thus we find that the singular double pole at order $1 / N^{2}$ is in a sense spurious, which appears because we took the large $N$ limit first, and it is recovered after the smooth average [1]. The density of state does not diverge for $E \rightarrow 0$. Recently the density of state of this complex matrix model has been studied in various contexts, and related expressions in terms of Bessel functions have been discussed [22].

We note that in the case of the Hermitian matrix model, the Bessel function is of half-integer order, and consequently we have no poles in the $1 / N$ expansion. The double pole $1 / x^{2}$ of the connected two-point correlation function cancels with a factor $\sin ^{2}(x)$ as $x \rightarrow 0$ [1]. Here we have a different situation since $\sin ^{2}\left(x-\frac{\pi}{4}\right)$ does not vanish for $x \rightarrow 0$. We must sum the leading pole terms of the equation above and it gives eventually a finite result for $E \rightarrow 0$ at fixed large $N$.

\section{Lattice of matrices}

We now extend our analysis to the general $d$-dimensional lattice of matrices [3]. We place $N$ by $N$ matrices on the lattice. The gluon propagator is given by $\sigma_{\alpha \beta}$ defined by

$$
\sigma_{\alpha \beta}=\frac{1}{M_{\alpha \beta}^{2}}
$$

Here $M_{\alpha \beta}$, as defined in [3], is a real symmetric matrix whose entries are the analogs of $m_{1}^{2}, m_{2}^{2}$, and $m^{2}$ in (2.6). The indices $\alpha$ and $\beta$, which label the lattice sites, run from 1 to $C$, where $C$ denotes the number of sites on the lattice. In leading order, the one-point Green's function is given by $G(z)=\frac{1}{N} \sum_{\alpha} g_{\alpha}$ where $g_{\alpha}$ is determined by

$$
g_{\alpha}=\frac{1}{E-\sum_{\beta} \sigma_{\alpha \beta} g_{\beta}}
$$

Let us restrict ourselves to a $d$-dimensional hypercubic lattice, on which a quantum particle hops with a hopping matrix is given by $\sigma$. We introduce $\varepsilon(k)$ by $[3]$

$$
\sigma_{\alpha \gamma}=\sum_{k}<\alpha|k>\epsilon(k)<k| \gamma>
$$


For the case of nearest neighbor hopping, we have

$$
\varepsilon(k)=\frac{1}{m^{2}}+\frac{2}{M^{2}} \sum_{a} \cos k_{a}
$$

Below we will calculate the one-point Green's function for the most general case with arbitrary $\varepsilon(k)$. For specific examples, we often take for simplicity the case $m^{2}=\infty$ and $M^{2}=2$.

We will now calculate the one-point Green's function to order $1 / N^{2}$. We consider here the general situation defined by some $\sigma$ matrix. In particular, for the simple example given in (2.5) we include the diagonal part of the Hamiltonian. The self-energy part to order $1 / N^{2}$ is obtained from the diagram of $D_{a}$ and $D_{b}$, where the number of rungs on the ladders are no longer restricted as they were before. We obtain

$$
\Sigma_{a}=G\left[\left(\frac{\sigma G^{2}}{1-\sigma G^{2}}\right)_{\alpha \alpha}\right]^{2}+G\left[\frac{\sigma^{2} G^{4}}{\left(1-\sigma G^{2}\right)^{2}}\right]_{\alpha \alpha}\left(\frac{\sigma G^{2}}{1-\sigma G^{2}}\right)_{\beta \beta}
$$

Note that repeated indices are not summed unless indicated otherwise. By translation invariance this expression is actually independent of $\alpha$ and $\beta$.

For the self-energy $\Sigma_{b}$, we have two parts $\Sigma_{b 1}$ and $\Sigma_{b 2}$. We obtain for $\Sigma_{b 1}$

$$
G \sum_{\gamma}\left(\sigma^{n}\right)_{\gamma \alpha}\left(\sigma^{m}\right)_{\alpha \gamma}\left(\sigma^{k}\right)_{\gamma \alpha} G^{2 n} G^{2 m} G^{2 k}=G \sum_{\gamma}\left[\left(\frac{\sigma G^{2}}{1-\sigma G^{2}}\right)_{\alpha \gamma}\right]^{3}
$$

where $n, m$ and $k$ are the number of the gluon propagators. The other part $\Sigma_{b 2}$, obtained by cutting one quark propagator inside the ladder, becomes

$$
\begin{aligned}
& G \sum_{\beta \gamma} \sum_{n, m, j, k}^{\infty}\left(\sigma^{m}\right)_{\beta \gamma}\left(\sigma^{n}\right)_{\gamma \alpha}\left(\sigma^{k}\right)_{\gamma \beta}\left(\sigma^{j}\right)_{\beta \alpha} G^{2 n} G^{2 m} G^{2 j} G^{2 k} \\
= & G \sum_{\beta \gamma}\left[\left(\frac{\sigma G^{2}}{1-\sigma G^{2}}\right)_{\beta \gamma}\right]^{2}\left(\frac{\sigma G^{2}}{1-\sigma G^{2}}\right)_{\alpha \gamma}\left(\frac{\sigma G^{2}}{1-\sigma G^{2}}\right)_{\alpha \beta}
\end{aligned}
$$

Note the rather unusual wasy in which the indices or site labels are arranged.

Let us check these expression for the case $C=2$ discussed earlier. We set

$$
\sigma=\left(\begin{array}{ll}
0 & 1 \\
1 & 0
\end{array}\right)
$$


The first term in (4.5) becomes

$$
\left(\frac{\sigma G^{2}}{1-\sigma G^{2}}\right)_{\alpha \alpha}=\frac{1}{2}\left(\frac{G^{2}}{1-G^{2}}-\frac{G^{2}}{1+G^{2}}\right)=\frac{G^{4}}{1-G^{4}}
$$

and

$$
\left(\left(\frac{\sigma G^{2}}{1-\sigma G^{2}}\right)^{2}\right)_{\alpha \alpha}=\frac{1}{2}\left(\frac{G^{4}}{\left(1-G^{2}\right)^{2}}+\frac{G^{4}}{\left(1+G^{2}\right)^{2}}\right)=\frac{G^{4}\left(1+G^{4}\right)}{\left(1-G^{4}\right)^{2}}
$$

The factor $1 / 2$ in (4.7) and (4.8) are necessary for a fixed $\alpha$ (which we do not sum over.) Thus we get the same result for the self-energy $\Sigma_{a}=2 G^{9} /\left(1-G^{4}\right)^{3}$ as was given earlier (2.11). As for $\Sigma_{b}$, we note

$$
\begin{aligned}
& \left(\sigma^{n}\right)_{\alpha \gamma}=\delta_{\alpha \gamma}(\mathrm{n}=\text { even }) \\
& \left(\sigma^{n}\right)_{\alpha \gamma}=\sigma_{\alpha \gamma}(\mathrm{n}=\text { odd })
\end{aligned}
$$

Using this property, we see that the numbers of gluon propagators $n, m, m$ or $n+j, m, k$ should be either all even or all odd. Thus we obtain the previous result for $\Sigma_{b}$ as given in (4.9) and (4.10).

We can now immediately go to a $d$-dimensional lattice on which matrices are placed by inserting the $\sigma$ given in (4.3). Thus, we obtain

$$
\begin{aligned}
\left(\frac{\sigma G^{2}}{1-\sigma G^{2}}\right)_{\alpha \beta} & =<\alpha\left|\frac{\sigma G^{2}}{1-\sigma G^{2}}\right| \beta> \\
& =\sum_{k}<\alpha|k><k| \frac{\sigma G^{2}}{1-\sigma G^{2}}|k><k| \beta> \\
& =\frac{1}{C} \sum_{k} e^{i k(\alpha-\beta)}\left(\frac{\varepsilon_{k} G^{2}}{1-\varepsilon_{k} G^{2}}\right)
\end{aligned}
$$

where we have used $\left\langle\alpha|k><k| \beta>=\frac{1}{C} e^{i k(\alpha-\beta)}\right.$. Using this diagonalized representation, we obtain for the different parts of the self-energy the following expressions:

$$
\begin{gathered}
\Sigma_{a 1}=G\left[\frac{1}{C} \sum_{k} \frac{\varepsilon_{k} G^{2}}{1-\varepsilon_{k} G^{2}}\right]^{2} \\
\Sigma_{a 2}=G\left(\frac{1}{C} \sum_{k} \frac{\varepsilon_{k}^{2} G^{4}}{\left(1-\varepsilon_{k} G^{2}\right)^{2}}\right)\left(\frac{1}{C} \sum_{p} \frac{\varepsilon_{p} G^{2}}{1-\varepsilon_{p} G^{2}}\right)
\end{gathered}
$$




$$
\begin{gathered}
\Sigma_{b 1}=G \sum_{\gamma}\left[\frac{1}{C} \sum_{k} e^{i k(\alpha-\gamma)}\left(\frac{\varepsilon_{k} G^{2}}{1-\varepsilon_{k} G^{2}}\right)\right]^{3} \\
\Sigma_{b 2}=G \sum_{\beta, \gamma}\left[\left(\frac{1}{C} \sum_{k} \frac{\varepsilon_{k} G^{2}}{1-\varepsilon_{k} G^{2}} e^{i k(\beta-\gamma)}\right)^{2}\left(\frac{1}{C} \sum_{q} \frac{\varepsilon_{q} G^{2}}{1-\varepsilon_{q} G^{2}} e^{i q(\alpha-\gamma)}\right)\right. \\
\left.\times\left(\frac{1}{C} \sum_{p} \frac{\varepsilon_{p} G^{2}}{1-\varepsilon_{p} G^{2}} e^{i p(\alpha-\beta)}\right)\right]
\end{gathered}
$$

where $C$ is the number of lattice points, $C=L_{1} \cdots L_{d}$. We note that by translation invariance $\Sigma_{b 1, b 2}$ are in fact independent of $\alpha$.

We can easily recover our previous results of course. For the two-site case we have $k=0$ and $k=\pi$, and $\beta, \gamma,=1,2$ with a fixed $\alpha=1$. For instance, noting that $\varepsilon_{k=0}=1$, and $\varepsilon_{k=\pi}=-1$, we get immediately

$$
\begin{aligned}
\Sigma_{b 1} & =\frac{G}{8} \sum_{\gamma=1}^{2}\left(\frac{G^{2}}{1-G^{2}}-e^{i \pi(1-\gamma)} \frac{G^{2}}{1+G^{2}}\right)^{3} \\
& =\frac{G^{7}\left(1+G^{6}\right)}{\left(1-G^{4}\right)^{3}}
\end{aligned}
$$

in agreement with(2.12). Similarly, we find easily that $\Sigma_{b 2}$ calculated here agrees with (2.13).

We have no divergence for the 3 -site lattice, where $k=0, \frac{2 \pi}{3}, \frac{4 \pi}{3}$ and $\varepsilon_{k}=1,-\frac{1}{2},-\frac{1}{2}$, respectively. Similarly for lattices with odd number of sites.

In contrast, for a one-dimensional lattice with the number of sites $C=$ $L=$ an even integer, we have $k=\pi$ and $\varepsilon_{k=\pi}=-1$, and thus we get a divergence when $G^{2}=-1$.

For $L \rightarrow \infty$, we find

$$
\begin{aligned}
\Sigma_{a 1} & =G\left[\frac{1}{C} \sum_{k} \frac{\cos (k) G^{2}}{1-\cos (k) G^{2}}\right]^{2} \\
& =G \frac{\left(1-\sqrt{1-G^{4}}\right)^{2}}{1-G^{4}} \\
\Sigma_{a 2}=G & \frac{\left(2 G^{4}-1+\left(1-G^{4}\right)^{3 / 2}\right)\left(1-\sqrt{1-G^{4}}\right)}{\left(1-G^{4}\right)^{2}}
\end{aligned}
$$


where $k=2 \pi(j-1) / L, j=1, \cdots L$ For the self-energy parts $\Sigma_{b 1}, \Sigma_{b 2}$, the sum over $\beta$ and $\gamma$ give complications. But the leading singularity of $\Sigma_{b 2}$ cancels exactly the singularity of $\Sigma_{a 2}$ in (4.20). The singularity of $\Sigma_{b 1}$ at $G^{2}=-1$ is the same as the singularity of $\Sigma_{a 1}$. Thus we determine the singularity for onedimensional case at the band center $G^{2}=-1$ with a single pole divergence in order $1 / N^{2}$. The cancellation of the leading singularity of $\Sigma_{b 2}$ with $\Sigma_{a 2}$ holds for any dimension and coincides with the cancellation found in ref.[5] for the lowest Landau level. We have also verified this cancellation by the numerical evaluation of the self-energy for large $L$ near $G^{2}=-1$.

The singularity $1 / N^{2}|E|$ in the density of state as $E \rightarrow 0$ is too strong, since the integral of the density of state should be equal to one. In one dimension, the off-diagonal disorder case of the tight binding model, in which the hopping matrix is real and $N=1$ case, is known to have a singularity of $1 /|E|(\ln |E|)^{3}$ in the density of state near $E=0$ [14]. In Fig.3, the density of state of a finite chain with real hopping matrix for $\mathrm{N}=1$, is evaluated for a box distribution. We consider the nearest neighbour random hopping, which is represented by the tridiagonal real matrix $M$,

$$
M=\left(\begin{array}{ccccc}
0 & b_{1}^{*} & 0 & \ldots & \\
b_{1} & 0 & b_{2}^{*} & 0 & \ldots \\
0 & b_{2} & 0 & b_{3}^{*} & \ldots \\
0 & 0 & b_{3} & 0 & b_{4}^{*} \\
& & \ldots & &
\end{array}\right)
$$

By the calculation of the density of the eigenvalue of this matrix, we obtain the curve of the density of state. The random variable $b_{i}$ is generated 5000 times, and the histogram of the eigenvalues is evaluated. From these calculation, we see the divergent singularity near $\mathrm{E}=0$. The singularity is consistent with $1 / E$ behavior, although it is difficult to see the existence of the logarithmic correction from this calculation.

For the complex hopping case, in which the coupling $b_{i}$ in (4.20) is a complex random number, the density of state shows oscillations similar to Fig.2. We evaluate this complex case in Fig. 4. The first peak near $E=0$ is finite for $L \rightarrow \infty$, where $L$ is the length of the chain.

It may be also interesting to note that we have the similar behavior in different examples. For the sparse random matrix, the density of state shows a singularity $1 /|E|(\ln |E|)^{2}$ instead of $1 /|E|(\ln |E|)^{3}[15]$. We have also another example, studied by Brézin, Gross and Itzykson who have obtained the 
same singularity $1 /|E|(\ln (|E|))^{2}$ in the density of state for the lowest Landau level with a random Poisson distribution [16].

For the two dimensional case, the self-energy $\Sigma_{a 1}$ behaves like $\left[\ln \left(1+G^{2}\right)\right]^{2}$ and $\Sigma_{a 2}$ like $\ln \left(1+G^{2}\right) /\left(1+G^{2}\right)$. This singularity is cancelled with a singularity in $\Sigma_{b 2}$. We find that the singularity of the self-energy is proportional to $\left[\ln \left(1+G^{2}\right)\right]^{2}$ near the band center. This coincides with the calculation of the two-site lowest Landau level result in ref. [5].

It is remarkable that we have the same singularity as in the two- state (spin up and down) degenerate quantum Hall case studied in [5]. We consider this a manifestation of the possibility that these two mdoels may same universality class. The integral of the density of state with this logarithmic square singularity is finite. The exact singularity of the off-diagonal tight binding model is not known. Numerical work [9] for the two-state lowest Landau level model also shows a singularity at the band center also. Moreover, the logarithmic square singularity seems to have an applicable region near $E=0$ according to a recent numerical study for the lowest Landau level, although the density of state does not diverge for $E \rightarrow 0$ [23]. The state of $E=0$ in the two-dimensional case is related to the zero energy wave function, for which the Atiyah-Singer index theorem can be applied. In this respect, one can perhaps relate the present problem to other interesting problems [24,25].

For two-dimensional case, we have also evaluated the density of state of the off-diagonal disorder $(\mathrm{N}=1)$. We examined the real and complex case similar to the one dimensional case. For the real case, the density of state seems divergent at $E=0$ and consistent with [26]. However, the density of state of the complex case, in which nearest neighbour hopping matrix element is complex, shows the similar behavior to the complex matrix model (Fig.2). The first peak near $E=0$ is finite.

\section{Discussion}

In this paper, we have discussed the singularity of the density of state in the large $N$ limit of a complex matrix model as well as its $d$-dimensional lattice generalization. We have found the singularity in order $1 / N^{2}$. We have compared our result with known results in $d=0$ and $d=1$. For $d=0$, the double pole singularity we obtained is reminiscent to the spurious double 
pole of the two-point correlation function in the Hermitian matrix model. For $d=1$, we compared our result with the off-diagonal tight binding model. Our result is different by the logarithmic factor. For $d=2$, we have obtained the logarithmic square singularity, which coincides with the result of [5] for the $N$-orbital two-state lowest Landau level quantum Hall model. We interprete this coincidence of the singularity as a manifestation of the possibility that these models belong to the same universality case. Although our calculation is restricted to order $1 / N^{2}$, the result of the singularity of the density of state gives a clue of what the true behavior might be. It may be interesting to evaluate the Green's functions to order $1 / N^{4}$. For the spin degenerate two-state quantum Hall system, the numerical similation suggests that there are three extended states [9]. In $1 / N$ expansion, the singularity $(\ln E)^{2} / N^{2}$ has been obtained for the density of state [5]. However, as we discussed in this paper, this is interpreted as the result after the smooth average. The density of state is considered to be finite for $E \rightarrow 0$. It is also important to note that the density of state has a nonvanishing value for $E \rightarrow 0$. Then, as discussed in [5], the conductivity is exactly given by $\sigma_{x x}=e^{2} / \pi h$ since the parameter $\theta$ in [5], defined by $\theta=-\tan ^{-1}(\operatorname{Im} G(z) / \operatorname{Re} G(z))$, becomes $-\pi / 2$.

\section{Acknowledgement}

We thank Edouard Brézin for discussions about the formulation of lattices of matrices. SH thanks K. Minakuchi for discussion of his numerical results. AZ would like to thank D. Arovas for mentioning that the lattices of matrices studied in [3] may be relevant for impurity scattering in spin-dependent quantum Hall systems. He would also like to thank M. Kohmoto for hispitality at the Institute for Solid State Physics, University of Tokyo, where this

work was initiated. This work is supported in part by the National Science Foundation under Grant No. PHY89-04035, and by CNRS-JSPS cooperative research project. 


\section{References}

[1] E. Brézin and A. Zee, Nucl. Phys. B40 (1993), 613.

[2] E. Brézin and A. Zee, Phys. Rev. E49 (1994) 2588.

[3] E. Brézin and A. Zee, "Lattice of matrices," Santa Barbara preprint (1994) NSF-ITP-94-75.

[4] E. Brézin, S. Hikami and A. Zee, Phys. Rev. E (1995), in press. (hepth.9412230).

[5] S. Hikami, M. Shirai and F. Wegner, Nucl. Phys. B408 (1993) 415.

[6] R. M. Gade, Nucl. Phys. B398 (1993), 499.

[7] D. K. K. Lee, Phys. Rev. B50 (1994), 7743.

[8] A. W. W. Ludwig, M. P. A. Fisher, R. Shanker and G. Grinstein, Phys. Rev. B50 (1994), 7526.

[9] C. B. Hanna, D. P. Arovas, K. Mullen and S. M. Girvin, a preprint (1994). cond-mat.9412102.

[10] F. J. Wegner, Phys. Rev. B19 (1979), 783.

[11] Yu. M. Makeenko, JETP-Lett. 52 (1990), 259.

J. Ambjørn, C. F. Kristjansen and Yu. M. Makeenko, Mod. Phys. Lett. A7 (1992), 3187.

[12] R. Oppermann and F. Wegner, Z. Phys. B34 (1979), 327.

[13] S. Hikami, Prog. Theor. Phys. 72 (1984), 722; Prog. Theor. Phys. 76 (1986), 1210.

[14] G. Theodorou and M. H. Cohen, Phys. Rev. B13 (1976), 4597.

T. P. Eggarter and R. Riedinger, Phys. Rev. B18 (1978), 569.

[15] J. Rodgers and C. De Dominicis, J. Phys. A23 (1990) 1567.

[16] E. Brézin, D. J. Gross and C. Itzykson, Nucl. Phys. B235 (1984), 24. 
[17] X. G. Wen and A. Zee, Phys. Rev. Lett. 69 (1992) 953, 3600(E), Phys. Rev. B47 ( 1993) 2265.

X. G. Wen and A. Zee," A phenomenological study of interlayer tunneling in double-layered quantum Hall systems", MIT-SBITP preprint.

[18] K. Yang, K. Moon, L. Zheng, A. H. MacDonard, S. M. Girvin, D. Yoshioka and S-C. Zhang, Phys. Rev. Lett. 72 (1994) 732.

[19] K. Slevin and T. Nagao, Phys. Rev. Lett. 70 (1993) 635.

[20] B. V. Bronk, J. Math. Phys. 6 (1965), 228.

[21] M. Abramowitz and I. A. Stegun, Handbook of Mathematical Functions, National Bureau of Standards, (1972) Washington, P.364.

[22] T. Nagao and K. Slevin, J. Math. Phys. 34 (1993) 2075.

A. V. Andreev, B. D. Simons and N. Taniguchi, Nucl. Phys. B432[FS] (1994) 487.

[23] K. Minakuchi, Master thesis, University of Tokyo (1995).

[24] X. G. Wen and A. Zee, Nucl. Phys. B316 (1989), 641.

[25] M. Inui, S. A. Trugman and E. Abrahams, Phys. Rev. B 49 (1994) 3190 .

[26] S. N. Evangelou, J. Phys. C 19 (1986), 4291. 


\section{Figure caption}

Fig.1a The diagrams $D_{a}$ and $D_{b}$ of order $1 / N^{2}$.

Fig.1b The self-energy $\Sigma_{a 1}, \Sigma_{a 2}, \Sigma_{b 1}$ and $\Sigma_{b 2}$, which are obtained from $D_{a}$ and $D_{b}$ by cutting the solid lines.

Fig.2 The density of state of the complex matrix model for $N=5$ (broken line) and $N=10$ (solid line). The semi-circle law $\sqrt{4-E^{2}} / \pi$ is also shown by a dotted line.

Fig.3 The density of state of a finite chain model $(\mathrm{L}=10)$ with off-diagonal disorder. The hopping random variable $b_{i}$ is real and obeys the box distribution with $-1<b_{i}<1$. There is a divergence at $E=0$.

Fig.4 The density of stae of a finite chain model $(\mathrm{L}=12)$ with off-diagonal disorder. The hopping random variable $b_{i}$ is complex. The real part and the imaginary part both obey the box distribution, which takes the value between -1 and 1 uniformly. 